\title{
Thicker endometrium on hCG trigger day improves the live birth rate of fresh cleavage embryo transfer in $\mathrm{GnRH}$-agonist regimen of normogonadotrophic women
}

\author{
Xi Luo ${ }^{1,2,3,4 \#}$, Yunxiu Li ${ }^{1,2 *}$, Haishan Zheng ${ }^{1,2}$, Lei Ding ${ }^{1,2}$, Manqin Zhang ${ }^{1,2}$, Yonggang $\mathrm{Li}^{1,2}, \mathrm{Ze} \mathrm{Wu}^{1,2}$ \\ ${ }^{1}$ Department of Reproductive Medicine, the First People's Hospital of Yunnan Province, Kunming, China; ${ }^{2}$ Reproductive Medical Center of Yunnan \\ Province, the Affiliated Hospital of Kunming University of Science and Technology, Kunming, China; ${ }^{3}$ Faculty of Life Science and Technology, \\ Kunming University of Science and Technology, Kunming, China; ${ }^{4}$ Medical School, Kunming University of Science and Technology, Kunming, China \\ Contributions: (I) Conception and design: X Luo; (II) Administrative support: Y Li, Z Wu; (III) Provision of study materials or patients: X Luo, Y Li; \\ (IV) Collection and assembly of data: H Zheng, L Ding, M Zhang; (V) Data analysis and interpretation: X Luo, Y Li, Y Li, Z Wu; (VI) Manuscript \\ writing: All authors; (VII) Final approval of manuscript: All authors. \\ \#These authors contributed equally to this work. \\ Correspondence to: Ze Wu. Department of Reproductive Medicine, the First People's Hospital of Yunnan Province, No 157 Jinbi Rd, Kunming \\ 650032, China. Email: wuzes2010@163.com; Yonggang Li. Department of Reproductive Medicine, the First People’s Hospital of Yunnan Province, \\ No 157 Jinbi Rd, Kunming 650032, China. Email: ygli232@vip.sina.com.
}

Background: Luteinizing hormone (LH) and progesterone (PROG) on human chorionic gonadotropin (hCG) trigger day are significantly correlated with assisted reproductive technology (ART) outcome. Moreover, LH and PROG are also involved in the functional preparation of the endometrium during the implantation window; however, whether they are related to endometrial thickness (EMT) is still unknown. The aim of the present study was to assess whether EMT has a positive correlation on the live birth rate following fresh embryo transfer (ET), and whether LH and PROG have an impact on EMT.

Methods: A total of 2,260 normogonadotrophic women were treated with a GnRH agonist for in vitro fertilization (IVF)/intracytoplasmic sperm injection. Patients with advanced age and poor ovarian reserve were excluded. The levels of LH, PROG, and EMT on the hCG trigger day were divided into binary variables, respectively, by the cutoff values, and which were obtained based on receiver operating characteristic curve analysis of live birth among LH, PROG and EMT levels on the hCG trigger day, respectively. Multivariate binary logistic regression was used to confirm the role of LH, PROG, and EMT on the live birth, and stratified analysis was used to determine whether LH and PROG have an impact on EMT.

Results: EMT and LH were protective factors for live births, with odds ratios (OR) of 1.11 [95\% confidence interval (CI): 1.066-1.157] and 1.696 (95\% CI: 1.345-2.139), respectively. However, PROG was a risk factor for live birth, with an OR of 0.635 (95\% CI: 0.526-0.766). The hierarchical cross-table analysis indicated that EMT had no significant difference for live birth in the combination of low LH and high PROG group. In the other subgroups, thick EMT was associated with a higher live birth rate $(\mathrm{P}<0.05)$.

Conclusions: On hCG trigger day, EMT, LH, and PROG all were independent factors that affected the live birth of fresh ETs. Thick EMT can significantly increase the live birth rate. However, multivariate logistic regression analysis showed that EMT does not affect the live birth rate in combination of low LH and high PROG environment.

Keywords: Live birth rate; luteinizing hormone (LH); endometrial thickness (EMT); progesterone (PROG); in vitro fertilization (IVF)

Submitted Mar 16, 2021. Accepted for publication May 06, 2021.

doi: $10.21037 / \mathrm{atm}-21-1922$

View this article at: http://dx.doi.org/10.21037/atm-21-1922

^ ORCID: Xi Luo, 0000-0002-9779-3697; Ze Wu, 0000-0002-8899-0445.

(C) Annals of Translational Medicine. All rights reserved. 


\section{Introduction}

In the process of human assisted reproductive technology (ART), there are many factors that affect the final live birth outcome, including ovarian reserve, ovarian responsiveness to exogenous gonadotropin (Gn), quality of the embryos, and maternal-fetal cross-talk during the implantation window (1-5). In the early stage of follicular development, endogenous reproductive hormone changes according to the addition of exogenous $\mathrm{Gn}$, and these changes have a significant impact on live birth outcomes (6).

In the existed studies, luteinizing hormone $(\mathrm{LH})$ and progesterone (PROG) on human chorionic gonadotropin (hCG) trigger day are significantly correlated with the outcome of ART in multiples studies, and are independent factors affecting the live birth $(7,8)$. Moreover, it has been confirmed that endogenous LH and PROG are also involved in the functional preparation of the endometrium during the implantation window period $(9,10)$. Although the specific mechanism is not clear, it is reasonable to speculate that LH and PROG on hCG trigger day may interact with the endometrium, thereby affecting the outcome. However, whether they are related to endometrial thickness (EMT) is still unknown, and whether EMT has a positive correlation on the live birth of fresh embryo transfers (ETs) is still unknown (11).

In the GnRH-agonist regimen, we analyzed the relationship between the live births of fresh ETs and indicators on the day of hCG trigger, such as LH, PROG, and EMT. Through subgroup cross-analysis, we clarified the clinical impact of LH and PROG on EMT. The findings provide new information for the preparation of the endometrium in future ART treatment process. We present the following article in accordance with the STROBE reporting checklist (available at http://dx.doi.org/10.21037/ atm-21-1922).

\section{Methods}

\section{Study population}

The present study was a retrospective study of patients who initiated their first ART cycle with the GnRHagonist regimen at the Reproductive Medicine Center of the First People's Hospital of Yunnan Province between January 1, 2017 and December 31, 2018. The present study was approved by the Medical Ethics Committee of the First People's Hospital of Yunnan Province (No.: KHLL2020-KY013), and the patients provided signed informed consent. All procedures performed in this study involving human participants were in accordance with the Declaration of Helsinki (as revised in 2013). All patients received conventional and standard in vitro fertilization (IVF)/intracytoplasmic sperm injection (ICSI) treatment without any additional intervention, and all treatments were conducted according to relevant guidelines and regulations.

Based on previous research, the premature elevation of PROG on the day of hCG trigger compromises ART outcome; therefore, for patients with PROG $\geq 2.5 \mathrm{ng} / \mathrm{mL}$ on the day of hCG trigger, fresh ET was cancelled, and frozen ET (FET) was used in a subsequent cycle. A total of 2,260 IVF/ICSI fresh ET cycles were ultimately included in our study.

\section{Inclusion criteria}

Patients who met the following criteria were included: controlled ovarian stimulation (COS) for the first time, GnRH-agonist regimen, age $<40$ years, number of oocytes retrieved $>3$, antral follicular count $(\mathrm{AFC}) \geq 5$, and antiMüllerian hormone $(\mathrm{AMH}) \geq 0.5 \mathrm{ng} / \mathrm{mL}$. There were available embryos on the third day of oocyte retrieval, and a fresh ET was completed. To exclude endogenous factors that may affect embryo quality or the endometrium, patients with poor ovarian response was excluded according to the Bologna consensus. Patients in whom the cause of infertility was limited to the fallopian tubes or male factors, and those with genetically related causes of infertility were also excluded.

\section{Ovarian stimulation and IVF/ICSI-ET}

The GnRH agonist was used to suppress the pituitary gland before stimulating the ovaries exogenously with recombinant follicle-stimulating hormone (FSH). During the mid-luteal phase of the menstrual cycle, $1.25-1.875 \mathrm{mg}$ of triptorelin acetate $(3.75 \mathrm{mg}$, Diphereline; Ipsen, Signes, France) was injected. After almost 14 days, transvaginal ultrasonography was performed, and serum estradiol (E2) concentrations were measured to confirm whether pituitary downregulation was complete. If there were no cysts $>2 \mathrm{~cm}$ in diameter and circulating $\mathrm{E} 2$ concentrations of $<50 \mathrm{pg} / \mathrm{mL}$, 150-300 IU of rFSH (75 IU, Gonal-F; Merck-Serono, Aubonne, Switzerland) or/and human menopausal gonadotropin (HMG) (75 IU, Lebaode; Livzon, Zhuhai, China) were administrated to initiate ovarian stimulation. The rFSH/HMG dose was adjusted according to the ovarian 
response throughout COS. When the average diameter of the 2 leading dominant follicles reached $18 \mathrm{~mm}$, EMT was evaluated by ultrasonographic examination and serum E2, LH, and PROG concentrations were tested, $250 \mu \mathrm{g}$ of recombinant hCG $(250 \mu \mathrm{g}$, Ovidrel; Merck-Serono, Modugno, Italy) was injected, and vaginal ultrasound-guided oocyte retrieval was performed 34-36 h later.

According to the condition of the spermatozoa, conventional IVF or ICSI was chosen. The number and morphology of blastomeres, and cellular debris within blastomeres were evaluated daily after fertilization. Fresh cleavage embryos were transferred on day 3 after oocyte retrieval, and the remaining high-quality embryos were cryopreserved for subsequent FET cycles. If there were more than 5 available embryos, blastocyst culture was recommended. To avoid complications caused by multiple pregnancies, and according to Chinese national regulations, as well as the patient's situation, the number of transferred embryos was not greater than 2. Luteal support was provided starting on the day of oocyte retrieval, using PROG sustained-release vaginal gel $(90 \mathrm{mg}$, Crinone; Merck-Serono, Hertfordshire, United Kingdom), PROG soft capsules (0.1 g, Utrogestan; Cyndea, Olvega, Spain), or dydrogesterone tablets (10 mg, Duphaston; Abbott, Olst, Netherlands), based on the patient's condition and according to the manufacturer's instructions. Serum $\beta$-hCG concentrations were tested on the 14th day, and if the pregnancy was positive, luteal support was continued for at least 8 weeks.

\section{Hormone measurements and clinical indicator definitions}

Serum hormone concentrations (i.e., AMH, FSH, PROG, LH, and E2) were measured by a BeckmanCoulter Unicel DxI 800 Access Analyzer (BeckmanCoulter, Brea, CA, USA). For all tests, the inter-assay coefficient of variation was less than $15 \%$, and the intraassay variation was less than $10 \%$. EMT was detected using the ACUSON NX3 Ultrasound System (Siemens, Munich, Germany).

$2 \mathrm{PN}$ was defined as the zygote with the presence of 2 pronuclei on the first day after oocyte retrieval; normal fertilization rate was defined as the ratio between the number of $2 \mathrm{PN}$ zygotes and the total number of oocytes retrieved; high-quality embryos referred to normal fertilization on day 1 , normal morphology and size on day 3 , total number of 6-10-blastomere embryos, and a cellular debris ratio $\leq 20 \%$; live birth referred to whether the fetus was alive after parturition.

\section{Statistical analysis}

Values were expressed as mean \pm standard deviation or median (interquartile range, 25 th-75th percentiles) according to the data distribution. Data comparison was performed by independent sample $t$-test or Mann-Whitney U-test according whether data normally distribution. Baseline data were all included in the multivariate logistic regression, and a forward stepwise likelihood ratio model was used. The receiver-operating characteristic (ROC) curve was used calculating for cutoff values. According to the cutoff value, the variable of LH, PROG, and EMT on the day of hCG trigger were divided into binary groups, respectively. An EMT greater than cutoff value constituted the thick EMT group, otherwise constituted the thin EMT group; LH or PROG greater than cutoff value was the high group, and below was the low group. Through the combination of $\mathrm{LH}$ and PROG groupings, stratified analysis was carried out to determine the role of EMT in each subgroup. All statistical analysis were performed by SPSS version 26 (IBM, Armonk, NY, USA). A 2-tailed $\mathrm{P}$ value of less than 0.05 was considered to be statistically significant.

\section{Results}

\section{Baseline analysis of live births}

A total of 2,260 fresh ET cycles were included in the present study, of which 844 were live births. The baseline data comparison of the live births found that the fertilization method and E2 on the day of hCG trigger had no significant impact on final outcome (Table 1). The EMT, LH, and PROG on the day of hCG trigger were found to have significant differences in the live birth group (Figure 1).

\section{Multivariate regression for live births of fresh ETs}

After the baseline analysis of live births, all variables were included in the multivariate logistic regression model. Table 2 summarizes the main independent factors affecting live births following fresh ETs. Of these, EMT, LH, and PROG on the day of hCG trigger were all independent factors affecting live births. EMT and LH were protective factors for live births, and their adjusted odds ratios (OR) were 1.11 [95\% confidence interval (CI): 1.066-1.157] and 1.696 (95\% CI: 1.345-2.139), respectively. However, PROG as a risk factor for live births, with an adjusted OR of 0.635 (95\% CI: 0.526-0.766). 
Table 1 Baseline analysis of live births following fresh embryo transfers

\begin{tabular}{|c|c|c|c|}
\hline Variables & Live births $(\mathrm{n}=844)$ & No live births $(n=1,416)$ & $P$ value \\
\hline BMI & $21.84 \pm 2.66$ & $22.23 \pm 2.71$ & 0.001 \\
\hline $\mathrm{AMH}(\mathrm{ng} / \mathrm{mL})$ & $3.39(2.19-5.07)$ & $3.17(2.11-4.71)$ & 0.019 \\
\hline AFC & $11(10-13)$ & $11(9-13)$ & $<0.001$ \\
\hline Dosage of Gn in COS (IU) & $2,825(2,300-3,425)$ & $3,050(2,450-3,725)$ & $<0.001$ \\
\hline \multicolumn{4}{|c|}{ Indicators on the day of hCG trigger } \\
\hline $\mathrm{LH}(\mathrm{mlU} / \mathrm{mL})$ & $0.70(0.52-0.94)$ & $0.60(0.45-0.84)$ & $<0.001$ \\
\hline E2 (pg/mL) & $2,942(2,020-3,914)$ & $2,896(1,949-3,864)$ & 0.240 \\
\hline Method for fertilization, $\mathrm{n}(\%)$ & & & 0.908 \\
\hline IVF & $682(80.8)$ & $1,147(81.0)$ & \\
\hline ICSI & $162(19.2)$ & $269(19.0)$ & \\
\hline No. oocytes retrieved & $12(9-15)$ & $11(8-15)$ & 0.001 \\
\hline Zygote $2 \mathrm{PN}$ rate & $0.67(0.50-0.80)$ & $0.62(0.44-0.76)$ & $<0.001$ \\
\hline No. embryos transferred, n (\%) & & & $<0.001$ \\
\hline 1 & $36(4.3)$ & $171(12.1)$ & \\
\hline 2 & $808(95.7)$ & $1,245(87.9)$ & \\
\hline
\end{tabular}

AFC, antral follicular count; AMH, anti-Müllerian hormone; BMI, body mass index; COS, control ovarian stimulation; EMT, endometrial thickness; E2, estradiol; hCG, human chorionic gonadotropin; ICSI, intracytoplasmic sperm injection; IVF, in vitro fertilization; LH, luteinizing hormone, PROG, progesterone, 2PN, 2 pronuclei.

\section{ROC analysis of EMT, LH, and PROG on live births}

In order to analyze whether there was an interaction among the indicators on hCG trigger day, the cutoff values of the 3 independent factors (EMT, LH and PROG) affecting live birth were obtained by ROC curve (Figure 2). EMT and LH were found to be positively correlated with live births, while PROG was inversely. The area under the curve (AUC) $(95 \% \mathrm{CI})$ and cutoff values were as follows: EMT: 0.571 (95\% CI: 0.547-0.595), 10.5 mm; LH: 0.578 (95\% CI: $0.554-0.603), 0.665 \mathrm{mIU} / \mathrm{mL}$; and PROG: 0.55 (95\% CI: $0.526-0.574), 1.455 \mathrm{ng} / \mathrm{mL}$. The three variables were divided into binary groups by cutoff values, respectively, and after the combination of LH and PROG groupings, the subgroup Stratified and multivariate analysis of EMT on live birth was performed.

\section{Stratified analysis of EMT on live births by $L H$ and PROG subgroups}

The hierarchical cross-table shows that there was no significant difference in the live births for EMT in the combination of low LH and high PROG group (Table 3), 

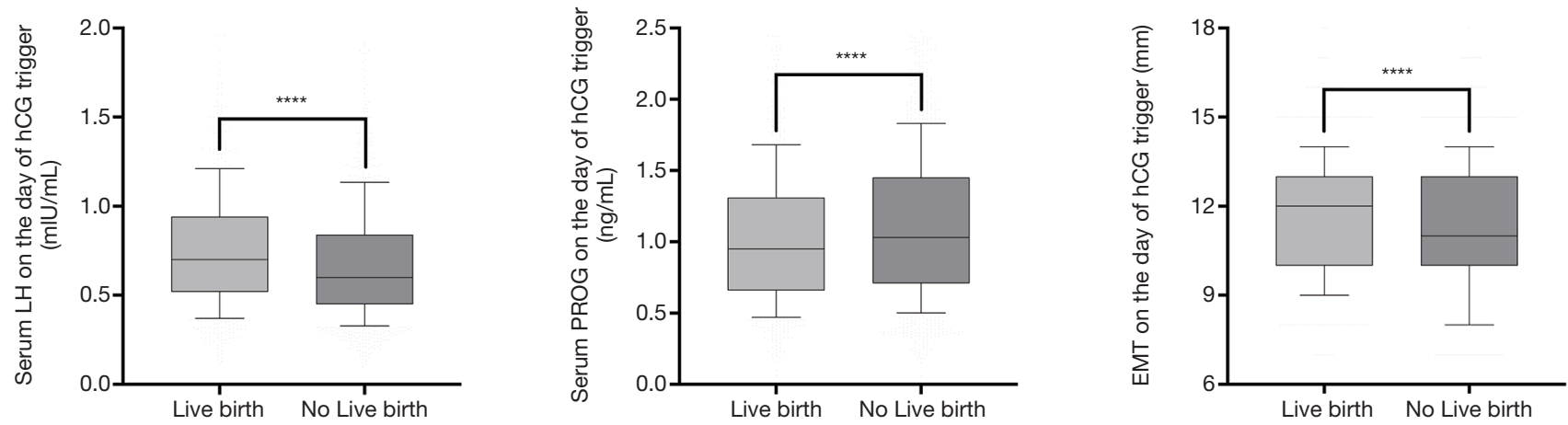

Figure 1 Box and whisker plot analysis of the luteinizing hormone (LH), progesterone (PROG), and endometrial thickness (EMT) on human chorionic gonadotropin (hCG) trigger day between the groups of live birth and no live birth. Box chart represents the 25th-75th percentiles. Middle line represents the median. whisker represent $10-90$ percentile. ${ }^{* * *} \mathrm{P}<0.0001$.

Table 2 Multivariable logistic regression of live births after fresh embryo transfer

\begin{tabular}{lccc}
\hline Variables & Adjusted OR & $95 \% \mathrm{Cl}$ & $\mathrm{P}$ value \\
\hline Age & 0.963 & $0.941-0.985$ & 0.001 \\
BMI & 0.958 & $0.926-0.991$ & 0.013 \\
EMT on hCG day & 1.110 & $1.066-1.157$ & $<0.001$ \\
LH on hCG day & 1.696 & $1.345-2.139$ & $<0.001$ \\
PROG on hCG day & 0.635 & $0.526-0.766$ & $<0.001$ \\
No. oocytes retrieved & 1.032 & $1.009-1.055$ & 0.006 \\
Zygote 2PN rate & 1.957 & $1.28-2.992$ & 0.002 \\
No. embryos transferred & 2.014 & $1.353-2.997$ & 0.001 \\
No. high-quality embryos transferred & 1.321 & $1.139-1.532$ & $<0.001$ \\
\hline
\end{tabular}

BMI, body mass index; Cl, confidence interval; EMT, endometrial thickness; hCG, human chorionic gonadotropin; LH, luteinizing hormone, OR, odds ratio; PROG, progesterone; 2PN, 2 pronuclei.
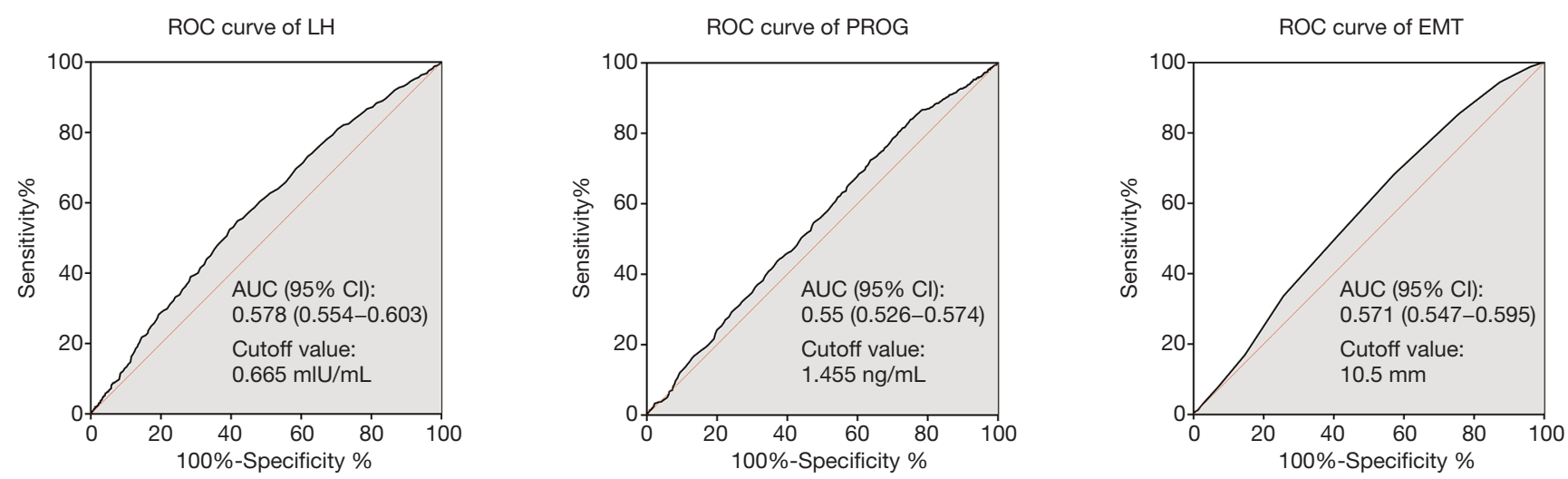

Figure 2 Correlation analysis between luteinizing hormone (LH), progesterone (PROG), or endometrial thickness (EMT) and live birth on the receiver-operating characteristic (ROC) curve. LH and EMT had a positive correlation with live birth, and PROG had a negative correlation. AUC, area under the curve; CI, confidence interval. 
Table 3 Hierarchical cross-table analysis of luteinizing hormone (LH) and progesterone (PROG) on live births in thin and thick endometrial thickness (EMT) groups

\begin{tabular}{|c|c|c|c|}
\hline Variables & Thin EMT & Thick EMT & $P$ value \\
\hline Low LH (n=261) & & & 0.140 \\
\hline Live births & $17(15.0)$ & $33(22.3)$ & \\
\hline No live births & $96(85.0)$ & $115(77.7)$ & \\
\hline Live births & $21(28.0)$ & $65(42.5)$ & \\
\hline No live births & $54(72.0)$ & $88(57.5)$ & \\
\hline \multicolumn{4}{|l|}{ Low PROG, n (\%) } \\
\hline Low LH (n=944) & & & 0.003 \\
\hline High LH ( $n=827), n(\%)$ & & & 0.004 \\
\hline Live births & $111(38.8)$ & 266 (49.2) & \\
\hline No live births & $175(61.2)$ & $275(50.8)$ & \\
\hline
\end{tabular}

Table 4 Multivariable logistic regression of endometrial thickness on live births in different luteinizing hormone (LH) or progesterone (PROG) groups

\begin{tabular}{lccc}
\hline Subgroups & Adjusted OR* & $95 \% \mathrm{Cl}$ & P value \\
\hline Low LH $(n=1,205)$ & 1.517 & $1.172-1.962$ & 0.002 \\
High LH $(n=1,055)$ & 1.516 & $1.162-1.980$ & 0.002 \\
Low PROG $(n=1,771)$ & 1.527 & $1.245-1.873$ & $<0.001$ \\
High PROG $(n=489)$ & 1.874 & $1.208-2.908$ & 0.005 \\
\hline
\end{tabular}

*, thick versus thin in EMT. LH, luteinizing hormone; PROG, progesterone; CI, confidence interval; OR, odds ratio; EMT, endometrial thickness.

and the $\mathrm{P}$ value is 0.14 . In other subgroups, Thick EMT was associated with a higher live birth rate $(\mathrm{P}<0.05)$.

\section{Multivariate regression of EMT in the combination of $L H$ and PROG subgroupings}

The multivariate logistic regression analysis in different combination subgroups was performed, and the model was adjusted by age, BMI, number of oocytes retrieved, zygote 2PN rate, number of embryos transferred, number of highquality embryos transferred. It was found that EMT was still an independent factor affecting the live birth in each subgroup (Table 4). However, after combining LH and PROG subgroups, especially, in the combination of low LH and high PROG group, the adjusted OR was 1.769 (95\% CI: 0.896-3.494, $\mathrm{P}=0.1$ ) (Table 5). Moreover, in combination of high PROG and high LH group, the $\mathrm{P}$ value was also close to the critical value. Figure 3 summarizes the adjusted OR value and $95 \%$ CI of EMT in the combination subgroups analysis. The results implied that in combination of low LH and high PROG group regardless of the thickness of the endometrium, it cannot promote live birth.

\section{Discussion}

The results of the present study showed that, among the indicators of hCG trigger day, EMT, LH, and PROG were independent factors that affected the live births following 
Table 5 Multivariable logistic regression of endometrial thickness on live births in different luteinizing hormone (LH) and progesterone (PROG) combined subgroups

\begin{tabular}{lccc}
\hline Subgroups & Adjusted $\mathrm{OR}^{*}$ & $95 \% \mathrm{Cl}$ & $\mathrm{P}$ value \\
\hline High PROG $(\mathrm{n}=489)$ & & & 0.100 \\
Low LH $(\mathrm{n}=261)$ & 1.769 & $0.896-3.494$ & 0.044 \\
High LH $(\mathrm{n}=228)$ & 1.864 & $1.016-3.420$ & \\
Low PROG $(\mathrm{n}=1,771)$ & & & 0.005 \\
Low LH $(\mathrm{n}=944)$ & 1.495 & $1.126-1.985$ & 0.013 \\
High LH $(\mathrm{n}=827)$ & 1.464 & $1.084-1.975$ & \\
\hline
\end{tabular}

*, thick versus thin in EMT. LH, luteinizing hormone; PROG, progesterone; Cl, confidence interval; OR, odds ratio; EMT, endometrial thickness.

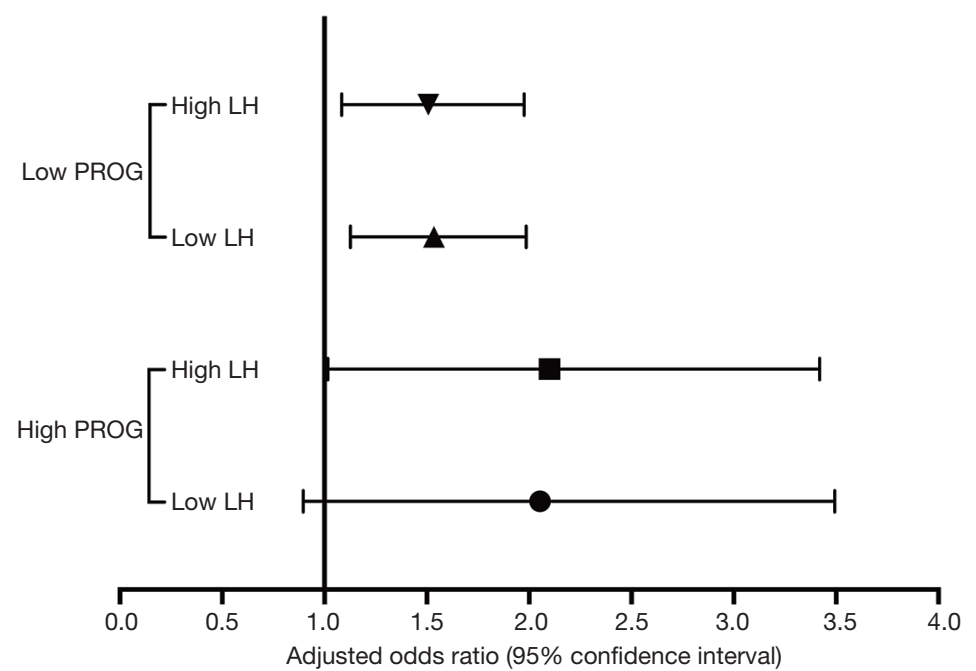

Figure 3 Adjusted odds ratio (95\% confidence interval) (thick versus thin in EMT) for live births in stratified groups of luteinizing hormones (LH) and progesterone (PROG) on human chorionic gonadotropin (hCG) trigger day.

fresh ETs. Thick EMT can significantly increase the live birth rate. However, multivariate logistic regression analysis showed that EMT does not affect the live birth in the combination of low LH and high PROG environments.

LH is believed to play an important role in follicular development and final maturation (12). High LH during the follicular phase may produce more mature oocytes, and is known to increase the live birth rate of fresh ET (13). In both agonist and antagonist regimens, studies have shown that high LH after COS has a beneficial effect on the ART outcome $(7,14,15)$; however, controversies still exist (16-18). Our results showed that high LH on hCG trigger day after COS was beneficial for live birth. There is a lack of research on the impact of LH on the outcome of fresh ETs, especially agonist regimens, and more cohort analyses are needed in future studies to explore the effects of $\mathrm{LH}$, other than promoting oocyte maturation during the periovulatory period. PROG is particularly important for late luteal support (19). It is generally believed that premature elevated PROG does not affect the quality of oocytes (9); however, it may lead to early offset of endometrial receptivity (8). Therefore, embryo implantation after transfer will be significantly compromised and can affect the cycle outcome $(8,20-25)$. Regarding the cause of premature PROG increase, some researchers believe that follicles exposed to high doses of FSH during COS will respond with an inappropriately high luteinizing hormone human chorionic gonadotropin receptor (LHCGR) expression. This in turn causes high PROG output in response to the trigger (24). EMT on 
the day of hCG trigger indicates the state of endometrial development. The thicker the EMT, the higher the possibility of live birth after fresh ET (26). Conversely, if the EMT is low on hCG trigger day, various complications may occur during pregnancy, which will also reduce the ART live birth rate (27-29).

Our findings indicated that, with low LH or high PROG alone, better EMT can also improve ART outcomes. However, with co-existing low LH and high PROG environment, although there EMT is thicker, the live birth rate following fresh ET will not improve. This implies that there is an interaction between LH, PROG, and that endometrium, that is, under the combined action of low LH and high PROG, the implantation condition of the endometrium was compromised. Despite a thick EMT, a better outcome cannot be achieved.

PROG has been found to directly act on the endometrium and participate in the embryo implantation process $(30,31)$. Moreover, the premature elevation of PROG on hCG trigger day was significantly related to a decrease in endometrial receptivity (32). Studies on the effect of LH on the endometrium are relatively scarce, but existing studies have found that LHCGR exists on the endometrium in animal models (33), and that its role is mainly to stimulate uterine growth, proliferation, and diastolic uterine movement (34). In human studies, a functional LHCGR on the endometrium has also been found $(35,36)$, which is regulated by the menstrual cycle and presents periodic changes. The expression of LHCGR reaches the highest level during the endometrial secretion phase (10), so it is reasonable to speculate that the low LH level following hCG trigger may affect the role of the endometrium during the implantation window. To sum up, it can be inferred that thick EMT cannot improve pregnancy outcomes under combination of low LH and high PROG condition on hCG trigger day.

Due to the inherent limitations of retrospective research, there was data selection bias in our study. Furthermore, some patients cancelled the fresh ET for various reasons, particularly when PROG was $>2.5 \mathrm{ng} / \mathrm{mL}$ on hCG trigger day, this may increase the data bias. If more accurate conclusions are to be drawn, further prospective studies are needed in the future to analyze the interaction mechanism between LH, PROG, and EMT on hCG trigger day and determine the reason for such results. In this way, we can better understand the role of reproductive hormone in the endometrial implantation window in order to improve the success rate of implantation.

\section{Acknowledgments}

Funding: This work was supported by the National Key Research and Development Program (Grant No. 2018YFC1002106), Program of Application and Fundamental Research of the Joint Special Project of Yunnan Provincial Science Technology Department \& Kunming Medical University [Grant No. 2018FE001(302)], Open Project of Yunnan Provincial Reproductive and Obstetrics and Gynecology Clinical Medicine Center (Grant No. 2019LCZXKF-SZ01), Key Projects of Basic and Applied Research in Yunnan Province (Grant No. 2018FA009), Yunnan Academic Leaders and Reserve Personnel (Grant No. 2017HB041), Medical Academic Leaders Training Program of Yunnan Province (D2017022), Program of Application and Fundamental Research of the Joint Special Project of Yunnan Provincial Science Technology Department \& Kunming Medical University [Grant No. 2018FE001(-120)], and the Yunnan Provincial Reproductive and Obstetrics and Gynecology Clinical Medicine Center (Grant No. zx2019-01-01).

\section{Footnote}

Reporting Checklist: The authors have completed the STROBE reporting checklist. Available at http://dx.doi. org/10.21037/atm-21-1922

Data Sharing Statement: Available at http://dx.doi. org/10.21037/atm-21-1922

Conflicts of Interest: All authors have completed the ICMJE uniform disclosure form (available at http://dx.doi. org/10.21037/atm-21-1922). The authors have no conflicts of interest to declare.

Ethical Statement: The authors are accountable for all aspects of the work in ensuring that questions related to the accuracy or integrity of any part of the work are appropriately investigated and resolved. All study designs were approved by the Ethics Committee of the First People's Hospital of Yunnan Province (No.: KHLL2020KY013), and the patients provided signed informed consent. All procedures performed in this study involving human participants were in accordance with the Declaration of Helsinki (as revised in 2013).

Open Access Statement: This is an Open Access article 
distributed in accordance with the Creative Commons Attribution-NonCommercial-NoDerivs 4.0 International License (CC BY-NC-ND 4.0), which permits the noncommercial replication and distribution of the article with the strict proviso that no changes or edits are made and the original work is properly cited (including links to both the formal publication through the relevant DOI and the license). See: https://creativecommons.org/licenses/by-nc-nd/4.0/.

\section{References}

1. Van Voorhis BJ. Outcomes from assisted reproductive technology. Obstet Gynecol 2006;107:183-200.

2. Hu S, Xu B, Jin L. Perinatal outcome in young patients with diminished ovarian reserve undergoing assisted reproductive technology. Fertil Steril 2020;114:118-24 e1.

3. Selcuk S, Bilgic BE, Kilicci C, et al. Comparison of ovarian responsiveness tests with outcome of assisted reproductive technology - a retrospective analysis. Arch Med Sci 2018;14:851-9.

4. Alpha Scientists in Reproductive M, Embryology ESIGo. The Istanbul consensus workshop on embryo assessment: proceedings of an expert meeting. Hum Reprod 2011;26:1270-83.

5. Weimar CH, Post Uiterweer ED, Teklenburg G, et al. In-vitro model systems for the study of human embryoendometrium interactions. Reprod Biomed Online 2013;27:461-76.

6. Andersen CY, Ezcurra D. Human steroidogenesis: implications for controlled ovarian stimulation with exogenous gonadotropins. Reprod Biol Endocrinol 2014;12:128

7. Benmachiche A, Benbouhedja S, Zoghmar A, et al. Low LH Level on the Day of GnRH Agonist Trigger Is Associated With Reduced Ongoing Pregnancy and Live Birth Rates and Increased Early Miscarriage Rates Following IVF/ICSI Treatment and Fresh Embryo Transfer. Front Endocrinol (Lausanne) 2019;10:639.

8. Esteves SC, Khastgir G, Shah J, et al. Association Between Progesterone Elevation on the Day of Human Chronic Gonadotropin Trigger and Pregnancy Outcomes After Fresh Embryo Transfer in In Vitro Fertilization/ Intracytoplasmic Sperm Injection Cycles. Front Endocrinol (Lausanne) 2018;9:201.

9. Kofinas JD, Mehr H, Ganguly N, et al. Is it the egg or the endometrium? Elevated progesterone on day of trigger is not associated with embryo ploidy nor decreased success rates in subsequent embryo transfer cycles. J Assist Reprod
Genet 2016;33:1169-74.

10. Licht P, von Wolff M, Berkholz A, et al. Evidence for cycle-dependent expression of full-length human chorionic gonadotropin/luteinizing hormone receptor mRNA in human endometrium and decidua. Fertil Steril 2003;79 Suppl 1:718-23.

11. Kasius A, Smit JG, Torrance HL, et al. Endometrial thickness and pregnancy rates after IVF: a systematic review and meta-analysis. Hum Reprod Update 2014;20:530-41.

12. Howles CM. Role of LH and FSH in ovarian function. Molecular and cellular endocrinology 2000;161:25-30.

13. Ferraretti AP, Gianaroli L, Magli MC, et al. Exogenous luteinizing hormone in controlled ovarian hyperstimulation for assisted reproduction techniques. Fertil Steril 2004;82:1521-6.

14. Westergaard LG, Laursen SB, Andersen CY. Increased risk of early pregnancy loss by profound suppression of luteinizing hormone during ovarian stimulation in normogonadotrophic women undergoing assisted reproduction. Hum Reprod 2000;15:1003-8.

15. Lahoud R, Al-Jefout $M$, Tyler J, et al. A relative reduction in mid-follicular LH concentrations during GnRH agonist IVF/ICSI cycles leads to lower live birth rates. Hum Reprod 2006;21:2645-9.

16. Cabrera RA, Stadtmauer L, Mayer JF, et al. Follicular phase serum levels of luteinizing hormone do not influence delivery rates in in vitro fertilization cycles down-regulated with a gonadotropin-releasing hormone agonist and stimulated with recombinant follicle-stimulating hormone. Fertil Steril 2005;83:42-8.

17. Esposito MA, Barnhart KT, Coutifaris C, et al. Role of periovulatory luteinizing hormone concentrations during assisted reproductive technology cycles stimulated exclusively with recombinant follicle-stimulating hormone. Fertil Steril 2001;75:519-24.

18. Razi MH, Mohseni F, Dehghani Firouzabadi R, et al. Results from adding recombinant $\mathrm{LH}$ for assisted reproductive technology treatment: A randomized control trial. Iran J Reprod Med 2014;12:111-6.

19. de Ziegler D, Pirtea P, Andersen CY, et al. Role of gonadotropin-releasing hormone agonists, human chorionic gonadotropin (hCG), progesterone, and estrogen in luteal phase support after hCG triggering, and when in pregnancy hormonal support can be stopped. Fertil Steril 2018;109:749-55.

20. Connell MT, Patounakis G, Healy MW, et al. Is the effect of premature elevated progesterone augmented by human 
chorionic gonadotropin versus gonadotropin-releasing hormone agonist trigger? Fertil Steril 2016;106:584-9.e1.

21. Tsai YR, Huang FJ, Lin PY, et al. Progesterone elevation on the day of human chorionic gonadotropin administration is not the only factor determining outcomes of in vitro fertilization. Fertil Steril 2015;103:106-11.

22. Horowitz E, Mizrachi Y, Finkelstein M, et al. A randomized controlled trial of vaginal progesterone for luteal phase support in modified natural cycle - frozen embryo transfer. Gynecol Endocrinol 2020:1-6.

23. Hu L, Xiong Y, Wang M, et al. Effect of progesterone on hCG day-to-basal progesterone ratio on live birth rate in long agonist fresh IVF/ICSI cycles: a 5-year, single-center study of more than 10,000 cycles. Gynecol Endocrinol 2021:1-5.

24. Friis Wang N, Skouby SO, Humaidan P, et al. Response to ovulation trigger is correlated to late follicular phase progesterone levels: A hypothesis explaining reduced reproductive outcomes caused by increased late follicular progesterone rise. Hum Reprod 2019;34:942-8.

25. Santos-Ribeiro S, Racca A, Roelens C, et al. Evaluating the benefit of measuring serum progesterone prior to the administration of HCG: effect of the duration of late-follicular elevated progesterone following ovarian stimulation on fresh embryo transfer live birth rates. Reprod Biomed Online 2019;38:647-54.

26. Holden EC, Dodge LE, Sneeringer R, et al. Thicker endometrial linings are associated with better IVF outcomes: a cohort of 6331 women. Hum Fertil (Camb) 2018;21:288-93.

27. He L, Zhang Z, Li H, et al. Correlation between endometrial thickness and perinatal outcome for pregnancies achieved through assisted reproduction technology. J Perinat Med 2019;48:16-20.

28. Moffat R, Beutler S, Schötzau A, et al. Endometrial

Cite this article as: Luo X, Li Y, Zheng H, Ding L, Zhang M, Li Y, Wu Z. Thicker endometrium on hCG trigger day improves the live birth rate of fresh cleavage embryo transfer in GnRH-agonist regimen of normogonadotrophic women. Ann Transl Med 2021;9(10):856. doi: 10.21037/atm-21-1922 thickness influences neonatal birth weight in pregnancies with obstetric complications achieved after fresh IVF-ICSI cycles. Arch Gynecol Obstet 2017;296:115-22.

29. Oron G, Hiersch L, Rona S, et al. Endometrial thickness of less than $7.5 \mathrm{~mm}$ is associated with obstetric complications in fresh IVF cycles: a retrospective cohort study. Reprod Biomed Online 2018;37:341-8.

30. DeMayo FJ, Lydon JP. 90 YEARS OF PROGESTERONE: New insights into progesterone receptor signaling in the endometrium required for embryo implantation. J Mol Endocrinol 2020;65:T1-t14.

31. Marquardt RM, Kim TH, Shin JH, et al. Progesterone and Estrogen Signaling in the Endometrium: What Goes Wrong in Endometriosis? Int J Mol Sci 2019;20:3822.

32. Lawrenz B, Fatemi HM. Effect of progesterone elevation in follicular phase of IVF-cycles on the endometrial receptivity. Reprod Biomed Online 2017;34:422-8.

33. Ziecik AJ, Stanchev PD, Tilton JE. Evidence for the presence of luteinizing hormone/human chorionic gonadotropin-binding sites in the porcine uterus. Endocrinology 1986;119:1159-63.

34. Ziecik AJ, Derecka K, Gawronska B, et al. Nongonadal LH/hCG receptors in pig: functional importance and parallels to human. Semin Reprod Med 2001;19:19-30.

35. Reshef E, Lei ZM, Rao CV, et al. The presence of gonadotropin receptors in nonpregnant human uterus, human placenta, fetal membranes, and decidua. The Journal of clinical endocrinology and metabolism 1990;70:421-30.

36. Bernardini L, Moretti-Rojas I, Brush M, et al. Status of hCG/LH receptor and $\mathrm{G}$ proteins in human endometrium during artificial cycles of hormone replacement therapy. J Soc Gynecol Investig 1995;2:630-5.

(English Language Editor: R. Scott) 